\title{
Electron-photon interaction in resonant tunneling diodes
}

\author{
Jesús Iñarrea, Ramón Aguado and Gloria Platero \\ Instituto de Ciencia de Materiales (CSIC), Cantoblanco, 28049 Madrid, Spain.
}

\begin{abstract}
We develope a model to describe the transmission coefficient and tunneling current in the presence of photon-electron coupling in a resonant diode. Our model takes into account multiphoton processes as well as the transitions between electronic states with different wave numbers. This is crutial to explain the experimental features observed in the tunneling current through a double barrier which cannot be reproduced with more simplified established models. According to our results, what experiments show in the current density are quantum photon-assisted features coming from multiphoton transitions which are not related with sample heating.
\end{abstract}

73.40.Gk 
Quantum transport through resonant heterostructures has been a very active research field in recent years, mainly due to the potential applicability of such devices, specially from a technological point of view. When an external electromagnetic field is connected to these semiconductor heterostructures, the physics interest and applications increase dramatically, for instance we can cite the case of photocounters, light detectors, microwave generators etc, In the last years a great effort has been done in theory [1 5] an experiments [6] 8] on transport in an AC field coupled to superlattices, double quantum wells and quantum dots. However much less work has been devoted to the case of an electromagnetic field irradiating a double barrier (DB), not only from a theoretical point of view but also from the experimental one [2.9] 13 . This last case, where the coupling to the continuum of states in the leads plays a crutial role in the current, has to be analized including the transitions between different wave number states caused by the electron-photon interaction [2, 10,11]. The most popular model for photoassisted tunneling (PAT) developed by Tien and Gordon (TG) [14] assumes that the interaction with the electromagnetic field is too weak to produce these transitions, i.e., the electron-photon interaction is treated as an efective potential $V_{i}$ within every spatial region $\mathrm{i}$ of the system. However, the exact coupling term in the Hamiltonian A.P ( $\mathrm{A}$ is the vector potential of the electromagnetic field and $\mathrm{P}$ the electronic momentum operator) produces transitions between different electronic states in the leads as well as in the quantum well. In DB's the coupling of the well states with the continuum at the leads produces a continuum of states in the well and the AC field induces transitions between them which modify the transmission coefficient and the tunneling current through the diode [2, 10]. Then a description in terms of the TG model which does not include those transitions is not suitable for describing PAT through a DB. However, in systems like multiple quantum wells, where the interwell sequential tunneling through quasidiscrete states determines the tunneling current, the TG model can be applied in most of the cases.

In this paper we calculate the coherent transmission coefficient and the current through a DB under the presence of light linearly polarized in the growth direction. For that purpose we develope a quantum mechanical formalism based in a canonical transformation and in time-dependent perturbation theory including multiphoton transitions. In the qualitative behaviour observed experimentally in the current density there are some features which at first sight do not seem to correspond to quantum PAT : the current threshold does not move linearly with the field frequency, as expected in quantum photoassisted tunneling. Also in the current density difference between the case with and without radiation as a function of the external bias, a peak shows up at a fixed bias, independent of the field frequency [13]. The present formalism gives good agreement with the experiments [13] and explains the observed features in the current in terms of quantum response, excluding a trivial heating effect from the laser pulse as responsible for them as well as classical rectification. The expression for the total Hamiltonian of the tunneling electron under the 
influence of a laser polarized in the growth direction $\mathrm{z}$ is given by:

$$
H_{t o t}=H_{e}^{0}+H_{p h}^{0}+W_{D}(t)+W_{O D}(t)
$$

where

$$
\begin{aligned}
H_{e}^{0} & =\sum_{k} \epsilon_{k} c_{k}^{+} c_{k} \\
H_{p h}^{0} & =\hbar w a^{+} a \\
W_{D}(t) & =\sum_{k}\left[\left(e / m^{*}\right)<k\left|P_{z}\right| k>c_{k}^{+} c_{k}(\hbar / 2 \epsilon V w)^{1 / 2}\left(a e^{-i w t}+a^{+} e^{i w t}\right)\right] \\
W_{O D}(t) & =\sum_{k} \sum_{k^{\prime} \neq k}\left[\left(e / m^{*}\right)<k^{\prime}\left|P_{z}\right| k>c_{k^{\prime}}^{+} c_{k}(\hbar / 2 \epsilon V w)^{1 / 2}\left(a e^{-i w t}+a^{+} e^{i w t}\right)\right]
\end{aligned}
$$

being $w$ the photon frequency. $H_{e}^{0}$ is the independent, electronic Hamiltonian and includes the double barrier potential and the external applied bias, therefore the eigenstates of $H_{e}^{0}, \Psi_{0}(k)$, are the tunneling states for bare electrons. $H_{p h}^{0}$, is the photon field Hamiltonian and $W_{D}$ and $W_{O D}$, describe the coupling between electrons and photons in the total Hamiltonian. Therefore the total Hamiltonian can be written as:

$$
H_{t o t}=H_{D}(t)+W_{O D}(t)
$$

where $H_{D}(t)=H_{e}^{0}+H_{p h}^{0}+W_{D}(t)$. The hamiltonian $H_{D}$, can be solved exactly considering a canonical transformation $U=e^{s}$ where $s=$ $\left(e / m^{*} \hbar w\right)(\hbar / 2 \epsilon V w)^{1 / 2}<k\left|P_{z}\right| k>c_{k}^{+} c_{k}\left(a^{+} e^{i \omega t}-a e^{-i \omega t}\right)$, obtaining an expression for the wave function of $H_{D}$ :

$$
\Psi_{D}(k)=\Psi_{0}(k) \sum_{n=-\infty}^{\infty} J_{n}\left(\beta_{k}\right) e^{-i n w t}
$$

$\mathrm{F}$ and $\omega$ are the electric field intensity and frequency of the laser field respectively, and $\beta_{k}=\frac{e F<P_{z}>}{m^{*} \hbar w^{2}}$. The dependence of the argument of the Bessel functions on the inverse of $\omega^{2}$ instead on the inverse of the frequency (as in TG model) comes from considering the quantum mechanical hamiltonian with the electron-photon coupling as in formula (4) and (5) (it can also be expressed in terms of the position operator). This dependence was already obtained in ref.10 (see also ref.2 ) and recently it was discussed in terms of scaling [12]. Once we have obtained the eigenstate for $H_{D}$, we apply time-dependent perturbation theory in order to treat the $W_{O D}(t)$ term. By doing this we have achieved an expression for the total wave function of the tunneling electron under the influence of a laser:

$$
\Psi(t)=\alpha\left[\Psi_{D}\left(k_{0}\right)+\sum_{m} b_{m}^{(1)}(t) \Psi_{D}\left(k_{m}\right)\right]
$$

denoting by $k_{0}$, the wave vector of the initial electron, and $k_{m}$ the wave vector of the corresponding electronic coupled states. The coefficients $b_{m}$ are given by: 


$$
b_{m}^{(1)}=\frac{-i e F L}{4 \hbar^{2} w} \sum_{n^{\prime}, n}\left[J_{n^{\prime}}\left(\beta_{k_{m}}\right) J_{n}\left(\beta_{k_{0}}\right) \frac{<k_{m}\left|P_{z}\right| k_{0}>}{k_{m}}\right]
$$

$n^{\prime}$ and $n$ run from $-\infty$ to $\infty$ and $m=n^{\prime}-n \pm 1= \pm 1, \pm 2, \pm 3, \ldots$. The normalization constant $\alpha=\frac{1}{\sqrt{1+\sum_{m}\left|b_{m}^{(1)}(t)\right|^{2}}}$, guarantees current conservation. $\Psi_{D}\left(k_{0}\right)$ is the "dressed" or diagonal reference state and $\Psi_{D}\left(k_{m}\right)$, represents the coupled "dressed" states due to photon absorption and emission. The spectral density associated to (8) consists in a central peak (weighted by $J_{0}^{2}$ ) and infinite n-sidebands separated in $n \hbar \omega$. from the central peak and weighted by $J_{n}$. If the argument of the Bessel functions is very small, the sidebands intensities are negligible and it is enough to consider transitions between the main side bands (the ones weighted by $J_{0}$ ) of different electronic states separated in energy by $\hbar \omega$. For higher values of the ratio: $\mathrm{F} / \omega^{2}$, the spectral density weight is shared between the satellite peaks and their contribution cannot be neglected. According to our formalism, the electronic eigenvalues are shifted in $\Delta=\frac{M^{2}}{\hbar w}$ being $M=\frac{e<P_{z}>}{m^{*}}\left(\frac{\hbar}{2 \epsilon V w}\right)^{1 / 2}$. This shift in energy which can be expressed in terms of $\mathrm{F}: M=\frac{e<P_{z}>}{m^{*}} \frac{F}{2 \sqrt{N} w}$ being $N$ the number of photons in the volume $V$, results to be negligible with respect to the electron eigenvalues 10,15 . Applying the current operator to the transmitted and incident wave function, we obtain the time-averaged coherent transmission coefficient following the Transfer Matrix technique:

$$
\begin{aligned}
T= & \frac{T_{0}}{\left(1+k_{1} / k_{0}\left|b_{1}^{(1)}\right|^{2}+k_{-1} / k_{0}\left|b_{-1}^{(1)}\right|^{2}+k_{2} / k_{0}\left|b_{2}^{(1)}\right|^{2}+k_{-2} / k_{0}\left|b_{-2}^{(1)}\right|^{2}+\ldots \ldots\right)}+ \\
& \frac{T_{1}\left|b_{1}^{(1)}\right|^{2}}{\left(k_{0} / k_{1}+\left|b_{1}^{(1)}\right|^{2}+k_{-1} / k_{1}\left|b_{-1}^{(1)}\right|^{2}+k_{2} / k_{1}\left|b_{2}^{(1)}\right|^{2}+k_{-2} / k_{1}\left|b_{-2}^{(1)}\right|^{2}+\ldots \ldots\right)}+ \\
& \frac{T_{-1}\left|b_{-1}^{(1)}\right|^{2}}{\left(k_{0} / k_{-1}+k_{1} / k_{-1}\left|b_{1}^{(1)}\right|^{2}+\left|b_{-1}^{(1)}\right|^{2}+k_{2} / k_{-1}\left|b_{2}^{(1)}\right|^{2}+k_{-2} / k_{-1}\left|b_{-2}^{(1)}\right|^{2}+\ldots \ldots\right)}+ \\
& \frac{T_{2}\left|b_{2}^{(1)}\right|^{2}}{\left(k_{0} / k_{2}+k_{1} / k_{2}\left|b_{1}^{(1)}\right|^{2}+k_{-1} / k_{2}\left|b_{-1}^{(1)}\right|^{2}+\left|b_{2}^{(1)}\right|^{2}+k_{-2} / k_{2}\left|b_{-2}^{(1)}\right|^{2}+\ldots \ldots\right)}+ \\
& \frac{T_{-2}\left|b_{-2}^{(1)}\right|^{2}}{\left(k_{0} / k_{-2}+k_{1} / k_{-2}\left|b_{1}^{(1)}\right|^{2}+k_{-1} / k_{-2}\left|b_{-1}^{(1)}\right|^{2}+k_{2} / k_{-2}\left|b_{2}^{(1)}\right|^{2}+\left|b_{-2}^{(1)}\right|^{2}+\ldots \ldots\right)}+ \\
& +\ldots . . .
\end{aligned}
$$

The results for the transmission coefficient (see figure 1) has been obtained for a $G a_{x} A l_{1-x} A s$ DB with well and barrier thicknesses of $50 \AA$, and for $\mathrm{F}=4.10^{5} \mathrm{~V} / \mathrm{m}$ and $\hbar \omega=13.6 \mathrm{meV}$. The carrier density is $n=10^{18} \mathrm{~cm}^{-3}$. The main features observed in the transmission coefficient, $\mathrm{T}(\mathrm{E})$, are multiple satellite peaks at both sides of the central one, coming from photon absorption and emission. The two closest peaks to the central one correspond to one photon processes, mainly to the transitions between the zero-side bands of electronic states differing in one photon energy. The transitions between higher side bands of different states differing in energy one photon have very 
low intensities for these parameters and give a very small contribution to these two peaks. The other two peaks separated $2 \hbar \omega$ from the main one correspond to processes involving two photons. Higher multiphoton transitions has a much weaker intensity.

In fig. 2(a) we have plotted $\mathrm{J} / \mathrm{V}$ in the presence of the FIR laser. The effect of the light on $\mathrm{J}$ can be observed in figures 2(b) and 2(c) corresponding to $F=4 \times 10^{5} \mathrm{~V} / \mathrm{m}$ for both cases and $\hbar \omega=13.6 \mathrm{meV}$ and $\hbar \omega=4.2 \mathrm{meV}$ respectively. In those figures we plot the current difference $\Delta J$ between the case where the light is present and the case where there is no light. One observes firstly that the current threshold takes place at the same bias, a main peak as well takes place for both cases at the same bias which corresponds to the bias for the current threshold without light. A shoulder appears for V close to the center of the current peak, a weak negative contribution shows up for higher bias and finally a small structure appears which is associated with the current cutoff. The fact that the current threshold takes place at lower bias than in the case without radiation is easy to understand: the electrons in the emitter absorb photons and the current flows for $E_{r}$ (energy of the resonant state in the well) separated $n \hbar \omega$ from the emitter Fermi energy. Then, regarding superficially to PAT theory, one would expect, at first sight, not only that the current threshold shift linearly with $\omega$ but also a dependence of the position in bias of the main peak in $\Delta J$ with $\omega$. However the experiments do not show this frequency dependence [13]. Some attempts have been made in order to explain those results, first in terms of classical response and secondly relating the experiments to sample heating due to the laser. The dependence of $\mathrm{J}$ with the temperature has been measured and shows qualitatively different behaviour than the obtained in the presence of the laser, therefore the heating does not explain the cited experiments [13]. Regarding the classical response, the photon energies considered are much larger than the energy broadening of the DB resonant state and a quantum behaviour is expected.

The agreement between the experimental PAT current and our results allows us to explain the features disscused above. The fact that the current threshold for different $\omega$ takes place at practically the same bias is related to the multiphotonic processes. Comparing in fig.2, the two cases corresponding to $\omega=13.6 \mathrm{meV}$ and $\omega=4.2 \mathrm{meV}$ for fixed $\mathrm{F}$, we observe that for the case of lower energy, as $\mathrm{F} / \omega^{2}$ is larger more side bands contribute efficiently to the current and therefore the $\mathrm{J}$ threshold moves to lower bias. In this case $\mathrm{n}$ side bands have a non-negligible contribution to $\mathrm{J}$ such that $\mathrm{nx} 4.2 \sim m \times 13.6$ where $\mathrm{m}$ is the number of side bands which support the current for $\hbar \omega=13.6 \mathrm{meV}(\mathrm{m}$ smaller than $\mathrm{n}$ ).

These multiphoton contributions wash out the linear dependence that the threshold bias should follow as a function of $\hbar \omega$ if only one photon process took place. The explanation for the same position in the main peak in $\Delta J$ is more subtle and it is related with the number of parallel states available to tunnel resonantly with the absorption of one or more photons. The main peak corresponds to the bias where the resonance energy, $E_{r}$, is just above 
the fermi energy, $E_{F}$, at the emitter. In this situation the number of parallel states which can tunnel resonantly via absorption of one or more photons is maximum no matter the $\omega$ we are considering. When $E_{r}$ crosses $E_{F}$ the absorption proccesses are now compensated by emission from the emitter states to $E_{r}$ and this fact reduces dramatically the general efficiency of the resonant assisted tunneling.

In conclusion, we explain for the first time all the features in the current through a DB induced by an electromagnetic field [13] by means of a theory which includes the mixing of electronic states with different wavevector by the external field and multiphoton transitions. Previous models which do not consider the mixing of states, are not suitable for explaining the PAT through a double barrier [14]. More elaborated models including this mixing but considering single photon proccesses [10] could not explain, in terms of PAT, the independence of the current threshold or cut-off with $\omega$.

Our present model explains the available data in terms of quantum response, excluding classical response and laser heating.

This work has been supported by the Comision Interministerial de Ciencia y Tecnologia of Spain under contract MAT 94-0982-c02-02. 


\section{REFERENCES}

1 J.Iñarrea and G.Platero, Europhys. Lett., 3443 (1996); J.Iñarrea and G.Platero, Europhys. Lett., 33, 477 (1996).

2 Ramón Aguado, Jesús Iñarrea and Gloria Platero, Phys.RevB, 5310030 (1996).

3 Mathias Wagner, Phys.Rev.B, 4916544 (1994).

$4 \quad$ Ned S.Wingreen, A.P.Jauho and Y.Meir, 488487 (1993).

$5 \quad$ Y.Dakhnovskii and H.Metiu, Phys.Rev.B, 514193 (1995).

$6 \quad$ L.P.Kouwenhoven, S.Jauhar, K.McCormick, D.Dixon and P.L.McEuen, 502019 (1994).

$7 \quad$ B.Keay,S.Zeuner, S.J.Allen,K.D.Maranowski, A.C.Gossard,U.Bhattacharya and M.J.W. Rodwell, Phys.Rev.Lett 754102 (1995).

8 J.S.Scott, J.P.Kaminski, M.Wanke, S.J.Allen, D.H.Chow, M.Lui and T.Y.Liu, Appl.Phys.Lett., 641995 (1994).

9 C.J.G.M.Langerak, B.N.Murdin, B.E.Cole, J.M.Chamberlain, M.Henini, M.Pate and G.Hill, Appl.Phys.Lett., 673453 (1995).

10 J.Inarrea, G.Platero and C.Tejedor, Phys.Rev.B, 504581 (1994).

11 J.Inarrea, and G.Platero, Phys.Rev.B, 515244 (1995).

12 M.Wagner, Phys.Rev.Lett., 764010 (1996).

13 V.A.Chitta, C.Kutter, R.E,M. de Bekker, J.C.Maan, S.J.Hawkworth, J.M.Chamberlain, M.Henini and G.Hill, J.Phys.: Condens. Matter., 63945 (1994); C. Kutter, Diplom Arbeit.

14 P.K.Tien and J.P.Gordon, Phys.Rev.B,129,647 (1963).

15 M.Jonson, Phys.Rev.B, 395924 (1989). 


\section{FIGURES}

FIG. 1. $\log _{10}$ of coherent T(E) through a DB (well and barriers $50 \AA$ wide). $\left(F=4 \times 10^{5} \mathrm{~V} / \mathrm{m}\right.$, $\hbar w=13.6 \mathrm{meV})$.

FIG. 2. a) Coherent current as a function of DC bias for a $\mathrm{Ga}_{.7} A l_{.3} A s \mathrm{DB}$ (well and barriers $50 \AA$ wide). b) $\Delta J / \mathrm{V}\left(\mathrm{F}=4 x 10^{5} \mathrm{~V} / \mathrm{m}, \hbar \omega=13.6 \mathrm{meV}\right)$. c) Same as in b) for $\left.\hbar \omega=4.2 \mathrm{meV}\right)$. 


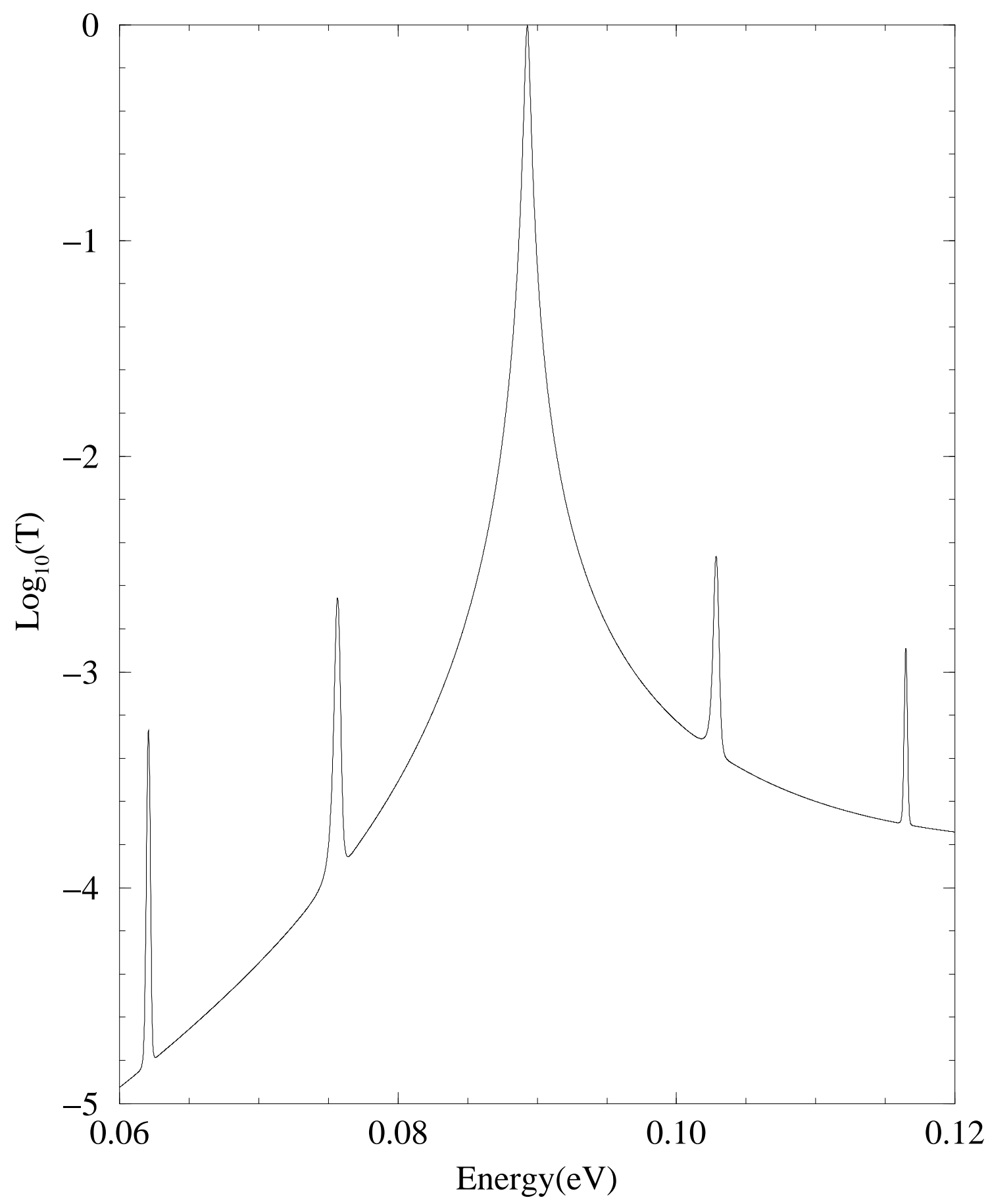




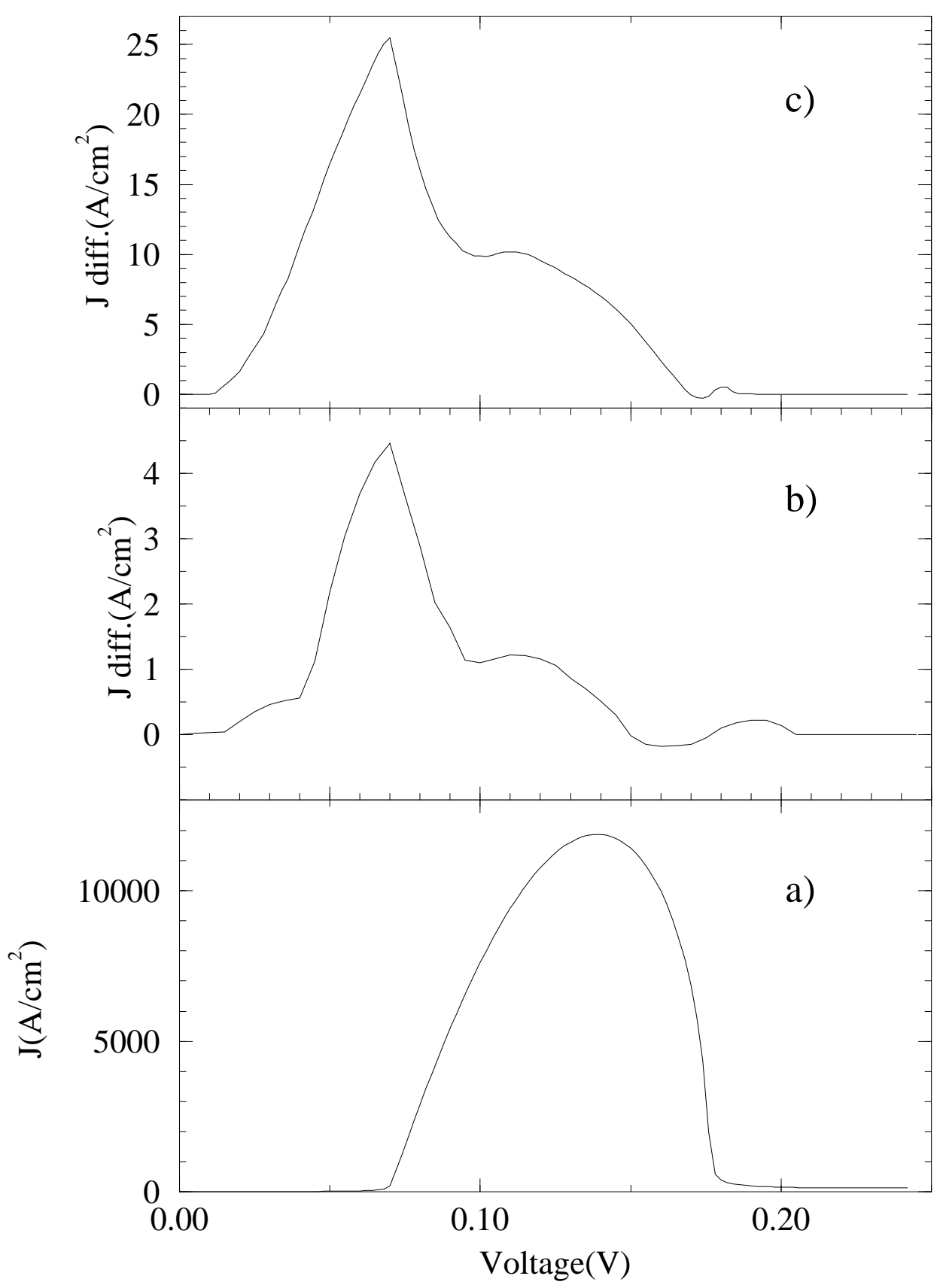

